\title{
Five-year experience of clinical ethics consultations in a pediatric teaching hospital
}

\author{
Streuli, Jürg C ; Staubli, Georg ; Pfändler-Poletti, Marlis ; Baumann-Hölzle, Ruth ; Ersch, Jörg
}

\begin{abstract}
Our retrospective study presents and evaluates clinical ethics consultations (CECs) in pediatrics as a structure for implementing hospital-wide ethics. We performed a descriptive and statistical analysis of clinical ethics decision making and its implementation in pediatric CECs at Zurich University Children's Hospital. Ninety-five CECs were held over 5 years for 80 patients. The care team reached a consensus treatment recommendation after one session in 75 consultations (89\%) and on 82 of 84 ethical issues $(98 \%)$ after two or more sessions (11 repeats). Fifty-seven CECs recommended limited treatment and 23 maximal treatment. Team recommendations were agreed outright by parents and/or patient in 59 of 73 consultations (81\%). Initial dissensus yielded to explanatory discussion or repeat CEC in seven consultations $(10 \%)$. In a further seven families $(10 \%)$, no solution was found within the CEC framework: five $(7 \%)$ required involvement of the child protection service, and in two families, the parents took their child elsewhere. Eventual team-parent/patient consensus was reached in 66 of 73 families (90\%) with documented parental/patient decisions (missing data, $\mathrm{n}=11$ ). Patient preference was assessable in ten CECs. Patient autonomy was part of the ethical dilemma in only three CECs. The Zurich clinical ethics structure produced a $98 \%$ intra-team consensus rate in 95 CECs and reduced initial team-parent dissensus from 21 to $10 \%$. Success depends closely on a standardized CEC protocol and an underlying institutional clinical ethics framework embodying a comprehensive set of transparently articulated values and opinions, with regular evaluation of decisions and their consequences for care teams and families.
\end{abstract}

DOI: https://doi.org/10.1007/s00431-013-2221-2

Posted at the Zurich Open Repository and Archive, University of Zurich

ZORA URL: https://doi.org/10.5167/uzh-105354

Journal Article

Published Version

Originally published at:

Streuli, Jürg C; Staubli, Georg; Pfändler-Poletti, Marlis; Baumann-Hölzle, Ruth; Ersch, Jörg (2014). Five-year experience of clinical ethics consultations in a pediatric teaching hospital. European Journal of Pediatrics, 173(5):629-636.

DOI: https://doi.org/10.1007/s00431-013-2221-2 


\title{
Five-year experience of clinical ethics consultations in a pediatric teaching hospital
}

\author{
Jürg C. Streuli • Georg Staubli • Marlis Pfändler-Poletti • \\ Ruth Baumann-Hölzle • Jörg Ersch
}

Received: 29 July 2013 / Accepted: 20 November 2013 /Published online: 10 December 2013

(C) Springer-Verlag Berlin Heidelberg 2013

\begin{abstract}
Our retrospective study presents and evaluates clinical ethics consultations (CECs) in pediatrics as a structure for implementing hospital-wide ethics. We performed a descriptive and statistical analysis of clinical ethics decision making and its implementation in pediatric CECs at Zurich University Children's Hospital. Ninety-five CECs were held over 5 years for 80 patients. The care team reached a consensus treatment recommendation after one session in 75 consultations ( $89 \%$ ) and on 82 of 84 ethical issues (98\%) after two or more sessions (11 repeats). Fifty-seven CECs recommended limited treatment and 23 maximal treatment. Team recommendations were agreed outright by parents and/or patient in 59 of 73 consultations $(81 \%)$. Initial dissensus yielded to explanatory discussion or repeat CEC in seven consultations (10\%). In a further seven families (10\%), no solution was found within the CEC framework: five (7\%) required involvement of the child protection service, and in two families, the parents took their child elsewhere. Eventual team-parent/patient consensus was reached in 66 of 73 families $(90 \%)$ with documented parental/patient decisions (missing data, $n=11$ ). Patient preference was assessable in ten CECs. Patient autonomy was part of the ethical dilemma in only three CECs. The Zurich clinical ethics structure produced a $98 \%$ intra-team consensus rate in $95 \mathrm{CECs}$ and reduced initial team-parent dissensus from 21 to $10 \%$. Success depends closely on a standardized CEC protocol and an underlying institutional clinical ethics framework embodying a comprehensive set of transparently articulated
\end{abstract}

J. C. Streuli $(\bowtie) \cdot$ G. Staubli $\cdot$ M. Pfändler-Poletti $\cdot$ J. Ersch Clinic of Pediatrics, University Children Hospital Zurich, Steinwiesstrasse 75, 8032 Zurich, Switzerland

e-mail: streuli@ethik.uzh.ch

R. Baumann-Hölzle

Interdisciplinary Institute for Ethics in Health Care, Dialog Ethik, Schaffhauserstrasse 418, 8050 Zurich, Switzerland values and opinions, with regular evaluation of decisions and their consequences for care teams and families.

Keywords Pediatric ethics $\cdot$ Parental authority $\cdot$ Best interests of the child $\cdot$ Decision making
Abbreviations
CEC Clinical ethics consultation
CPS Child protection service
DNR Do not resuscitate
QALY Quality-adjusted life year

\section{Introduction}

High-quality pediatrics requires high-quality ethics. Decisions on complex physiological and psychological issues within the therapeutic triangle of child, parents, and experts raise challenging ethical questions $[2,4,6,14,31]$. Insufficient reflection on, and communication of, values and facts can result in frustration, stress, and unsatisfactory decision making [20, 21].

Clinical ethics consultations (CECs) aim to tackle critical individual problems in a timely fashion, clarify the relevant medical facts, identify the ethical core issues (along with any other sources of conflict), and place them in a systemic valueand principle-grounded context. They then develop appropriate courses of action with due regard to the ethical and legal setting. The final step consists of well-documented implementation involving all stakeholders and meticulous follow-up $[10,19,22,26]$.

CECs are interventions with side effects and interactions. Although widely used, they have prompted few studies quantifying their contribution to clinical practice. The purpose of the present study, undertaken in a Swiss university children's hospital that had devised a dedicated clinical ethics structure 
one decade previously, was to review all clinical ethics consultations held over a 5-year period in order to determine whether ethical decision making could be further improved and, if so, how. We also propose further research and followup studies to develop better quality standards for the clinical implementation of clinical ethics services.

\section{Methods}

Background

An early model of clinical ethics consultations in pediatrics was developed in 1994 at the University Hospital of Zurich. CECs take place within an overriding Ethics Forum framework designed to foster an ethical dimension to clinical practice [10]. The core group in an Ethics Forum consists of 13 interdisciplinary healthcare professionals meeting on a regular six-weekly basis under the guidance and/or supervision of a medical ethicist. Working in a manner similar to an institutional review board, their responsibilities include education, advanced training, consultation debriefing, drafting of guidelines and recommendation templates, and the conduct and oversight of the CECs that are themselves but one part of an overarching ethics structure designed to establish an institution-wide ethics culture.

The CECs themselves proceed according to a predefined multistep model facilitating moral inquiry, deliberation, and consensus. Their aim, on an individual healthcare issue, is to come up with a recommendation that has been developed and endorsed by the whole team, patient, and/or parents. At the same time, they are designed to facilitate implementation of the recommendation by communicating, mediating, and continuously reviewing information exchange within the therapeutic triangle over the longer term. The format is that of a 60 - to 90 -min round table that can be - other than the core group - convened ad hoc within $48 \mathrm{~h}$, potentially triggered by all professionals, patients, and parents confronted with an ethical question. The meeting follows two slightly different multistep models based on the two sets of environments and needs in our hospital: a seven-step open-outcome general pediatric model and a more structured intensive care model focused on maximal versus limited treatment (Fig. 1). Both include a moderator (provided by the core group) and professionals directly involved in the patient's care (inner circle) as well as experts, including a representative of the department head, who are relevant but not directly involved (outer circle). Both models require a consensus from the inner circle. The outer circle's function is advisory, while the department head's representative retains a power of veto (Fig. 2). The number of participants during a CEC varies from 6 to 14 persons, depending on the number of disciplines and team members involved. Basically, every professional who can contribute information concerning the associated problems and facts is invited. Once the care team (inner circle) reaches a consensus

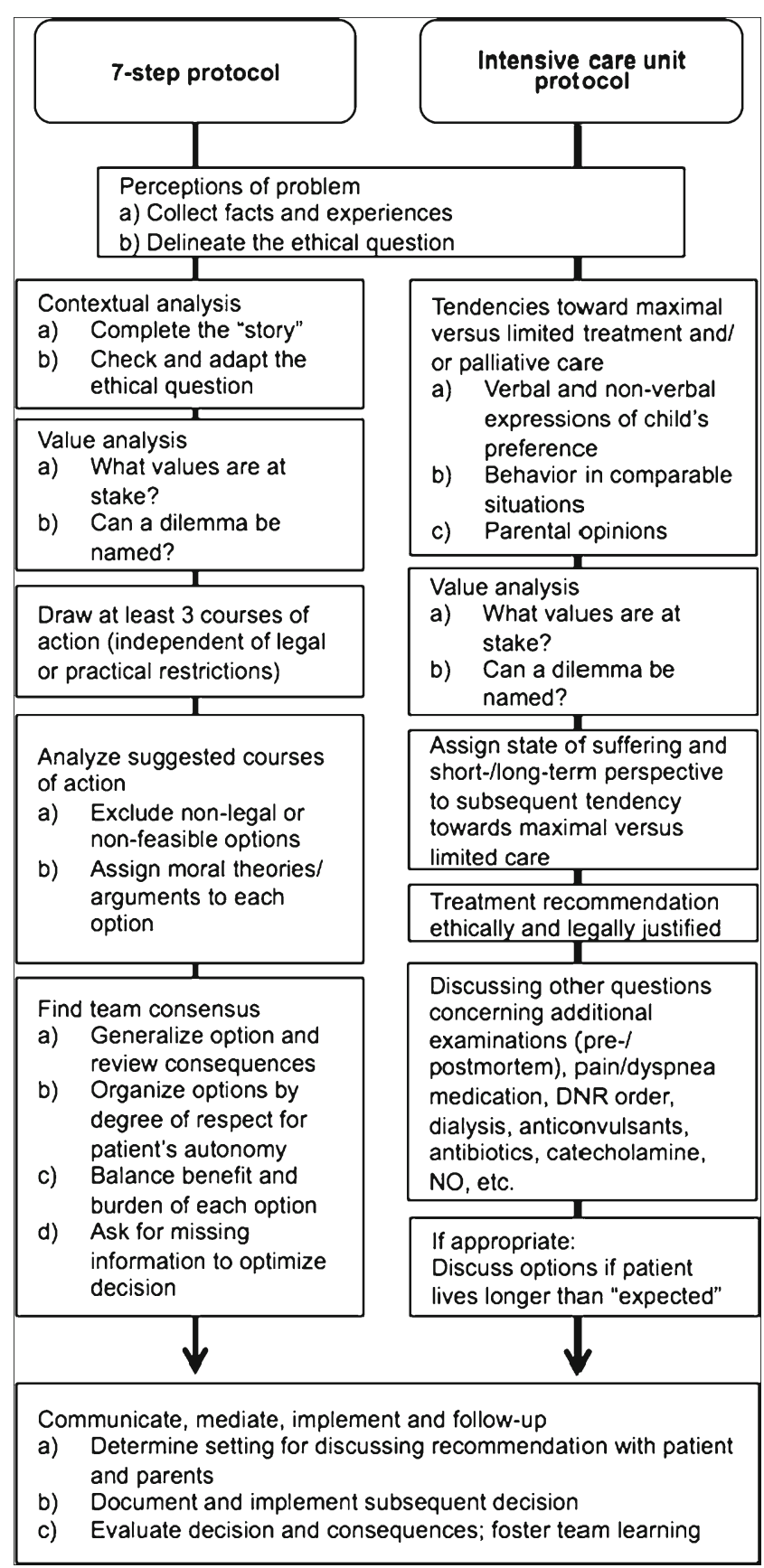

Fig. 1 Zurich models of pediatric ethical decision making

on a recommendation, it is a central task of a CEC to clarify how and by whom the resulting recommendation will be presented to the parents and/or the patient. In case of disagreement, the care team will repeat a CEC. The care team then has to find a new consensus, which may result in the acceptance of a split decision or in the involvement of the child protection service $[8,9]$.

Study population

We examined the records of all CECs conducted between 1 January 2006 and 31 December 2010 at the largest children's 
Fig. 2 Components of the ethics forum and composition of the inner and outer circles in the Zurich clinical ethics consultation model

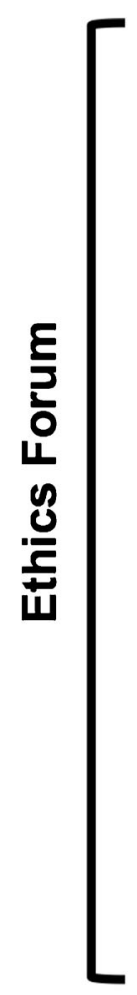

hospital in Switzerland. In 2010, the hospital had 218 beds (medicine, $n=72$; surgery, $n=60$; intensive care/neonatology, $n=39$; rehabilitation, $n=47$ ) occupied by 6,889 patients over 66,228 inpatient days (average stay, 9.6 days; average bed occupancy, $87 \%$ ).

Study instruments

Patient/parent characteristics, problems, preferences, and principles; CEC recommendations; and, if available, the patient's subsequent progress and outcome were tabulated in Excel 2010 (Microsoft Corporation, Redmond, WA) and analyzed using SPSS (version 19.0.0.1, IBM Company, Armonk, NY). The information was anonymized prior to tabulation. Inquiries and consultations conducted outside the CEC setting, e.g., at the bedside, by telephone, or in the core group, were not included.

\section{Results}

Patient characteristics

There is a slight but not significant male preponderance (56\%). Twelve patients had more than one CEC, at intervals of a few days to several months, for the same or a new ethical

Core group

(education, advanced training, case debriefing, drafting of guideline and recommendation templates, And conduct and oversight of CECs)

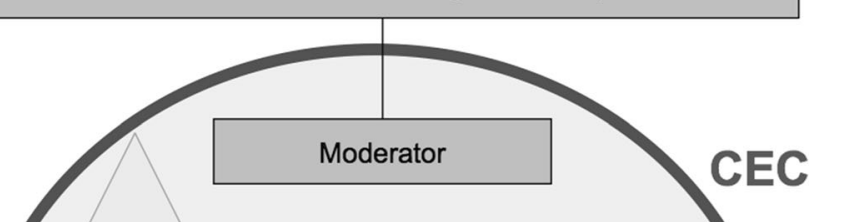

Outer circle Affiliated experts

Inner circle Patient care team

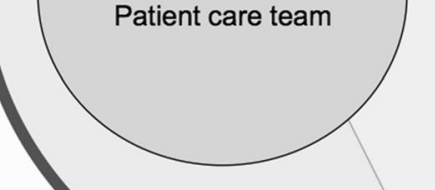


Table 1 Demographic, clinical, and administrative characteristics of the 80 patients discussed at the 95 clinical ethics consultations ( 15 repeats) at Zurich University Children's Hospital between 1 January 2006 and 31 December 2010

\begin{tabular}{|c|c|}
\hline & $n(\%)$ \\
\hline $\operatorname{Sex}(\mathrm{M} / \mathrm{F})$ of patients $(n=80)$ & $45 / 35(56 / 44)$ \\
\hline \multicolumn{2}{|l|}{ Age at CEC $(n=95)$} \\
\hline Newborn $<4$ weeks & $16(17)$ \\
\hline Baby $<1$ year & $34(36)$ \\
\hline Child $1-5$ years & $14(15)$ \\
\hline Child 6-12 years & $15(16)$ \\
\hline Adolescent $>13$ years & $16(17)$ \\
\hline \multicolumn{2}{|l|}{ Prognosis $(n=95)$} \\
\hline Precarious/diminished QALY & $23(24)$ \\
\hline Poor/severely diminished QALY & $67(71)$ \\
\hline Terminal & $5(5)$ \\
\hline \multicolumn{2}{|l|}{ Diagnosis $(n=95)$} \\
\hline Congenital syndrome & $32(34)$ \\
\hline Perinatal brain injury & $14(15)$ \\
\hline Cardiopathy & $12(13)$ \\
\hline Progressive CNS disease & $7(7)$ \\
\hline Severe infection & $7(7)$ \\
\hline Malignancy & $5(5)$ \\
\hline Multi-organ failure & $5(5)$ \\
\hline Traumatic brain injury & $5(5)$ \\
\hline Immunological problem & $2(2)$ \\
\hline Inborn error of metabolism & $2(2)$ \\
\hline Lung disease & $2(2)$ \\
\hline Tissue disease and bone & $2(2)$ \\
\hline \multicolumn{2}{|l|}{ Departments } \\
\hline ICU & $58(61)$ \\
\hline Rehab & $13(14)$ \\
\hline Oncology & $4(4)$ \\
\hline Bone marrow transplantation & $4(4)$ \\
\hline Nephrology & $3(3)$ \\
\hline Neonatology & $3(3)$ \\
\hline Immunology & $2(2)$ \\
\hline Respiratory medicine & $2(2)$ \\
\hline Mixed units & $2(2)$ \\
\hline Surgery & $2(2)$ \\
\hline Outpatient setting & $2(2)$ \\
\hline
\end{tabular}

$C N S$ central nervous system, $I C U$ intensive care unit, $Q A L Y$ qualityadjusted life year

"Do not resuscitate" (DNR) order. In 79 sessions, at least one secondary issue was present, mainly conflict within the treatment team $(n=21)$, projected quality of life $(n=20)$, treatment appropriateness, and treatment goals $(n=18$; Table 2$)$.

In one third of CECs $(n=31)$, parent preference was for maximal treatment; in 13 sessions, the parents opted for as
Table 2 Contents and issues debated at 95 clinical ethics consultations (15 repeats) at Zurich University Children's Hospital between 1 January 2006 and 31 December 2010

$n(\%)$

Main issue

Withdrawing or withholding treatment

$42(44)$

Appropriateness of treatment, goals of care

$34(36)$

Resuscitation issues ("do not resuscitate" orders)

$11(12)$

Quality of life

Allocation of resources

$1(1)$

Disagreement team-parents

$1(1)$

Legal-ethics interface

$1(1)$

Patient autonomy

$1(1)$

Family conflict

$1(1)$

Staff or professional conflict

Secondary issue

Disagreement team-parents $21(22)$

Quality of life $20(21)$

Appropriateness of treatment, goals of care, futility 18 (19)

Withdrawing or withholding treatment 5 (5)

Resuscitation issues ("do not resuscitate" orders) 4 (4)

Family conflict 4 (4)

Staff or professional conflict 3 (3)

Allocation of resources 3 (3)

Patient autonomy 1 (1)

No secondary issue $\quad 16(17)$

Parents' preference

Maximal treatment 31 (33)

"Healthcare professionals should decide what's best" $19(20)$

Not to harm, no suffering 13 (14)

Unsure or discordant 10 (11)

Minimal treatment 6 (6)

More intervention than team, using alternative medicine 2 (2)

Palliative care 2 (2)

Letting child die 1 (1)

No data 11 (12)

Patient's preference

No data 85 (89)

"Get treatment, get healthy" 3 (3)

Wants no further treatment 2 (2)

Unable to form an authentic preference 2 (2)

In accordance with the team 1 (1)

Wants to be with parents 1 (1)

Will be asked after the meeting 1 (1)

Dilemma

Beneficence/non-maleficence $\quad 88$ (93)

Autonomy/beneficence 2 (2)

Beneficence/distributive justice 1 (1)

Autonomy/distributive justice 1 (1)

No dilemma described 3 (3) 
little suffering as possible for their child. Twelve parents wanted to have the healthcare professionals to decide for them and ten parents were unsure of or in disagreement about their own preferences. There was no correlation between patient preference and native or non-native Swiss background (Pearson's $\chi^{2}=1.14, d f=1, P=0.29$ ).

Parent preference, indication of wishes, and expression of opinion were documented in 84 of all CECs. Patient preference was assessable and documented in ten CECs. Patient autonomy (the right to hold views, to make choices, and to take actions based on personal values and beliefs) was part of the dilemma in three consultations. Conflict between the principles of beneficence (the positive requirement of providing benefits to someone) and non-maleficence (the negative prohibition of actions or influences causing harm) was by far the most preponderant ethical dilemma (93\%).

\section{Recommendations and outcome}

CEC recommendations limited curative treatment in 57 of 95 consultations (palliative/comfort care, $n=26$; DNR order, $n=$ 17 ; submaximal optimized treatment, $n=9$; withdrawal of lifesaving treatment, $n=4)$. Maximal treatment was recommended in 23 consultations (Table 3).

The inner circle achieved a consensus after one session on 75 of 84 different issues $(89 \%)$ and after two sessions on seven issues $(8 \%)$. In two patients, there was no second session after dissensus due to the patient's early death.

Fifty-nine parents $(81 \%)$ agreed outright with the team recommendation. Seven families $(10 \%)$ initially dissented before consenting after explanatory discussion or one further CEC $(n=6)$ or three further CECs $(n=1)$. CECs failed to reach a solution in seven other families (10\%): five families (7\%) required the involvement of the child protection service, and in two families ( $3 \%$ ), the parents took their child elsewhere with no legal repercussions. Follow-up on the basis of the available medical history was only possible in $45(56 \%)$ of 85 patients: 29 (64\%) died before discharge, 5 (11\%) died shortly after discharge, and 11 (24\%) were alive at data assessment. The power of veto by the department head's representative has not been used in any case.

\section{Discussion}

We present the Zurich Ethics Forum approach to structuring a hospital-wide ethics culture and offer detailed statistical analysis of its implementation in 95 consecutive pediatric clinical ethics consultations. Care team consensus reached $98 \%$ and parent/child-team consensus $90 \%$.

To our knowledge, our paper offers the largest retrospective analysis of CECs in the pediatric literature. In comparison to
Table 3 Resolution and outcome of the 95 clinical ethics consultations (15 repeats) at Zurich University Children's Hospital between 1 January 2006 and 31 December 2010

\begin{tabular}{|c|c|}
\hline & $n(\%)$ \\
\hline \multicolumn{2}{|l|}{ Resulting recommendation $(N=95)$} \\
\hline Full treatment & $23(24)$ \\
\hline Differentiated treatment & $57(60)$ \\
\hline Palliative care & $26(27)$ \\
\hline "Do not resuscitate" order & $17(18)$ \\
\hline Optimized submaximal treatment & $9(10)$ \\
\hline Withdraw life-saving treatment & $4(4)$ \\
\hline Differentiated resuscitation & $1(1)$ \\
\hline Other & $13(14)$ \\
\hline Talking with patient & $5(5)$ \\
\hline Collecting more information & $2(2)$ \\
\hline Talking with parents & $2(2)$ \\
\hline Child protection service & $1(1)$ \\
\hline Optimized treatment & $1(1)$ \\
\hline Exploring new options & $1(1)$ \\
\hline No resulting recommendation & $3(3)$ \\
\hline \multicolumn{2}{|l|}{ Time to team consensus } \\
\hline One meeting & $75(89)$ \\
\hline Two meetings & $7(8)$ \\
\hline Dissensus without new meeting & $2(2)$ \\
\hline Total excluding repeats $(n=11)$ & $84(100)$ \\
\hline \multicolumn{2}{|l|}{ Time to team-parent/patient consensus } \\
\hline One meeting & $59(81)$ \\
\hline Two meetings & $6(8)$ \\
\hline Four meetings & $1(1)$ \\
\hline Dissensus and involvement of child protection service & $5(7)$ \\
\hline Dissensus and removal of child from hospital & $2(3)$ \\
\hline Total excluding repeats $(n=11)$ and missing data $(n=11)$ & $73(100)$ \\
\hline \multicolumn{2}{|l|}{ Follow-up } \\
\hline Died in hospital & $29(64)$ \\
\hline Alive according to last entry & $11(24)$ \\
\hline Reported death shortly after discharge & $5(11)$ \\
\hline Total excluding repeats $(n=15)$ and missing data $(n=35)$ & $45(100)$ \\
\hline
\end{tabular}

our 19 formal consultations per year, a survey in the USA from 1999 reported, among 275 general hospital committees, a mean of 8.1 formal consultations for both adults and children per year, with a median of 4 [27]. A German evaluation from 2002 to 2008 reported roughly three pediatric consultations per year [28]. Higher numbers were reported by a clinic of neonatology concerning exclusively end-of-life decisions in a uniform setting (e.g., 30 formal ethic meetings per year) using an earlier protocol of the Zurich Models of Pediatric Ethical Decision-Making [5]. Basically, hospitals have to balance a high number of formal ethic meetings against greater efforts, while higher numbers of consultations do not necessarily lead 
to higher quality of ethical decision making. In our opinion, ethical decision making is a responsible process of cogent reflection leading to a weighty decision, which cannot be outsourced to a committee or an ethician. To keep the process of decision making, the resulting decision, and its implementation close to all persons involved, a hospital must commit a certain amount of its scarce resources. According to subsequent qualitative interviews (data not shown here), most medical professionals think that 20 CECs per year in our hospital is a reasonable number. This certainly does not mean that the number could and should not be increased. However, the discussion is bound to further reflections about resource allocation and the importance of clinical ethics-a debate that our data can trigger, but not resolve.

Nevertheless, given individual needs and resources, the question arises of why and when a CEC is requested. DuVal [20] identified the most common factors triggering physicians' requests: (1) the need for help in resolving a conflict; (2) the need for assistance in interacting with a difficult family; (3) the need for help in making a decision or planning care; and (4) emotional triggers, such as intimidation, fear, or frustration. They concluded that these factors must be clearly identified so they can be properly addressed. However, our results suggest that the trigger does not have to be identical with the underlying ethical problem, nor does it have to play a central role in decision making. On the contrary, our findings suggest that intra-team and team-family conflicts exist in most consultations and - according to DuVal - may be fundamental in triggering a CEC. However, they were only of secondary interest in identifying the ethical problem and reaching a consensus about a solution. Given the very low number of team or team-parent conflicts discussed (team or team-parent dissensus was reported as a main issue in a single $\mathrm{CEC}$ ) and the high rate of resulting consensus, we assume that the consensus culture in combination with moral inquiry and mediation based on human dignity and human rights does not only respect and absorb intrapersonal conflicts but also shifts the focus onto the ethical problem beneath.

However, the use of a closely structured model as presented here is controversial itself, mostly on the grounds that it reduces complex ethical issues to mediation practicalities where outcome is determined by preset team opinions based on hierarchy, culture, or reflex societal values [1, 7, 13, 29]. Theoretically, these are important objections, but the evidence in their favor is slight or nonexistent. To our knowledge, there are no data indicating that the outcomes of consensus-centered decisions are ethically or practically worse or that in-depth ethical inquiry by an ethician would be a better way of reaching a "right and proper" decision in clinical practice. However, there are clearly limits to every consensus culture. Although an intra-team consensus rate of $98 \%$ on complex ethical questions can be seen as a positive outcome [2], it remains an open question whether the Zurich model offers the right balance between given structures and unbiased, ethically sound reflection.

Although we see prior team consensus as a key precondition for constructive discussion with the patient and/or parents, we also see the risk of inappropriate paternalism in a preconceived team opinion. Parent or caregiver tended in at least 19 consultations to place a decision in the hands of professionals by asking them to decide the best for their child. Moreover, parent preference was undocumented in 11 consultations. Behind these numbers, we assume a combination of simple documentation shortfall and parents overwhelmed by feelings of helplessness, ambivalence, anxiety, or guilt [33]. In our experience, there is a thin line between rash and unjustified handover of parental responsibility and overstraining the (evolving) capacities of parents to reach decisions in the tremendously difficult and unanticipated situations that occur on an ICU. At least one survey reported no evidence of parent inclusion in $27 \%$ of pediatric ethics consultations, but the extent to which parental preference was considered was unclear [25]. To safeguard the patient or surrogate decision maker from potential paternalism, the CEC's duty must be to document the patient's and/or caregiver's values along with any subsequent conflict within the therapeutic triangle. Any recourse to the weight of a healthcare professional recommendation against, or instead of, an (initial) parental preference, and/or inclusion of the child protection service, should be justified in transparent detail (e.g., parental denial of blood transfusion or medication instead of moderate to good prognosis). Moreover, as parents might recognize a moral dilemma differing from that perceived by health professionals, CECs should offer repeated consultation if a moral dilemma is solved from the perspective of the team, but not from that of the parent or child [30]. Therefore, the influence of different clinical ethics approaches on the therapeutic triangle can and should be subject to continuous review, comparison, and critical reflection.

The realization that children not only can but also must be included in the decision-making process has gained increased acceptance in recent years $[3,12,15,16,18]$. Common ground can be found by agreeing the child's status as a subject, which implies the requirement for adequate protection and provision, and the consideration of the child's volition, with due regard for its corresponding and developing abilities [17, 24, 32]. Our sample contained mainly neonates, infants, and young children (62\%), many children in intensive care (60\%), and many critically ill children ( $72 \%)$, who are rarely responsive. Given the very few children and adolescents involved actively in decision making ( $3 \%$ ), we are currently evaluating the use of age-appropriate participation facilitators such as expression of wish forms, computer questionnaires, diaries, and patient information documents, as proposed by Fraser et al. [23]. Whether thorough documentation of verbal and nonverbal expression of volition will include severely ill 
children in a more active and comprehensive manner remains to be shown in further evaluation and research.

There can be no single conclusive answer to what constitutes "good ethical practice" in pediatrics. What is needed instead is a vigorous and constructive analysis of clinical ethics on different levels, including empirical data from patients, parents, and professionals, backed by theoretical reflection on norms, concepts, and methodologies. To create common ground, we propose pooling the clinical ethics requirements formulated by field leaders and working parties. For every CEC, we recommend the use of a comprehensive protocol covering the issue(s) at stake, the preferences expressed by the child and/or parents, the principles underlying the dilemma, the consensus recommendation, and the family's reaction to that recommendation. Moreover, clinical ethics should entail continued education, evaluation, and quality management, designed to establish a practice-oriented culture of ethical reflection. Since bioethics emerged as a discipline in the early 1970s, it has been trying hard to translate ethical theory into clinical practice. Unfortunately, however, theory, once refined and simplified, becomes-or remainslargely unhelpful for specific clinical problems [11]. Both theory and practice must recognize their limitations and interdependence, and so far, no theory has been proven superior for clinical problems, and no form of clinical practice can claim impeccability. If excellence in clinical ethics is possible at all, ethical decision making should focus on a thorough integration of facts and values with practice-oriented recommendations and continuously evaluated exchange between all stakeholders. We hope that our data will foster constructive discussion on different approaches to these challenges.

\section{Conclusion}

Over the 5-year study period, transparent and comprehensible discussion achieved an intra-team consensus on treatment recommendations in $98 \%$ of 95 ethically and clinically complex CECs and reduced parent-team dissensus from an initial 21 to $10 \%$. CECs lessen the tension between individual life designs and therapeutic or care options, but cannot fully eliminate it. Compromise is inevitable in the clinical application of ethics, with continuous evaluation of process, structure, theory, and quality at all levels within an organization offering the only safeguard, along with regular welldocumented follow-up. In particular, we see four ways to ensure and to improve the quality of ethical decision making. Firstly, the process of decision making should be subject to complete and comprehensive documentation, including the values of parents, patients, and professionals. Secondly, if anyone in the therapeutic triangle does not communicate its preference, circumstances and reasons should be documented and reflected as well. Thirdly, CECs are interventions with possible side effects and should undergo follow-up research, including its impact on families and professionals. And fourthly, the available resources and the number of CECs should be actively matched and critically reflected. The Zurich model is only one of many options for ethical decision making, and it goes without saying that willingness to critically question decisions and actions remains an absolute prerequisite for implementing an ethics culture within an organization and a great challenge for everyone involved. With our data, we hope to give impetus for further research on the implementation of clinical ethics and its effects on our patients, their families, and the involved professionals.

\section{Funding source No funding sources.}

Financial disclosure The authors have no financial relationships relevant to this article to disclose.

Conflict of interests All authors are or were employed by the organization studied here. No sponsoring has been received for the research, and the authors do not have any conflict of interests.

\section{References}

1. Adams DM, Winslade WJ (2011) Consensus, clinical decision making, and unsettled cases. J Clin Ethics 22:310-327

2. Akre V, Falkum E, Hoftvedt BO, Aasland OG (1997) The communication atmosphere between physician colleagues: competitive perfectionism or supportive dialogue? A Norwegian study. Soc Sci Med 44:519-526

3. Alderson P, Sutcliffe K, Curtis K (2006) Children as partners with adults in their medical care. Arch Dis Child 91:300-303

4. American Academy of Pediatrics (2003) Family pediatrics: report of the task force on the family. Pediatrics 111:1541-1571

5. Arlettaz R, Mieth D, Bucher H-U, Duc G, Fauchère J-C (2005) Endof-life decisions in delivery room and neonatal intensive care unit. Acta Paediatr 94:1626-1631

6. Aulisio MP, Arnold RM, Youngner SJ (2000) Health care ethics consultation: nature, goals, and competencies: a position paper from the Society for Health and Human Values-Society for Bioethics Consultation Task Force on Standards for Bioethics Consultation. Ann Intern Med 133:59-69

7. Aulisio MR (2011) "Facilitated consensus," "ethics facilitation," and unsettled cases. J Clin Ethics 22:345-353, author reply 358-362

8. Baumann-Hölzle R (2009) "7 Schritte Dialog"-Exemplarische Vertiefung der Methodik einer Fallbesprechung. In: BaumannHölzle R, Arn C (eds) Ethiktransfer in Organisationen. EMH/ Schwabe, Basel, pp 215-266

9. Baumann-Hölzle R, Waldvogel K, Staubli G, Maguire C, Bänziger O, Huber Y, Sennhauser F (2009) Implementierung - "7 Schritte dialog" im Rahmen des Ethik-forums am Kinderspital Zürich. In: Baumann-Hölzle R, Arn C (eds) Ethiktransfer in Organisationen. EMH/Schwabe, Basel, pp 255-266

10. Baumann-Hölzle R, Maffezzoni M, Bucher H (2005) A framework for ethical decision making in neonatal intensive care. Acta Paediatr 94:1777-1783 
11. Beauchamp TL (2004) Does ethical theory have a future in bioethics? J Law Med Ethics 32:209-217

12. Brandazzi GF (2008) The European Association for Children in Hospital (EACH) Charter. Pediatrics 121:S97

13. Casarett DJ, Daskal F, Lantos J (1998) The authority of the clinical ethicist. Hastings Cent Rep 28:6-11

14. Committee on Bioethics (2001) Institutional ethics committees. Pediatrics 107:205-209

15. Committee on Bioethics (2007) Professionalism in pediatrics: statement of principles. Pediatrics 120:895-897

16. Committee on Hospital Care and Institute for Patient- and FamilyCentered Care (2012) Patient- and family-centered care and the pediatrician's role. Pediatrics 129:394-404

17. Cummings CL, Mercurio MR (2010) Ethics for the pediatrician: autonomy, beneficence, and rights. Pediatr Rev 31:252-255

18. De Lourdes Levy M, Larcher V, Kurz R, Ethics Working Group of the Confederation of European Specialists in Paediatrics (CESP) (2003) Informed consent/assent in children. Statement of the Ethics Working Group of the Confederation of European Specialists in Paediatrics (CESP). Eur J Pediatr 162:629 633

19. Dubler NN, Webber MP, Swiderski DM (2009) Charting the future. Credentialing, privileging, quality, and evaluation in clinical ethics consultation. Hastings Cent Rep 39:23-33

20. DuVal G (2001) What triggers requests for ethics consultations? J Med Ethics 27:24-29

21. Forde R, Vandvik I (2005) Clinical ethics, information, and communication: review of 31 cases from a clinical ethics committee. J Med Ethics 31:73-77
22. Forrow L, Arnold RM, Frader J (1991) Teaching clinical ethics in the residency years: preparing competent professionals. J Med Philos 16: 93-112

23. Fraser J, Harris N, Berringer AJ, Prescott H, Finlay F (2010) Advanced care planning in children with life-limiting conditionsthe wishes document. Arch Dis Child 95:79-82

24. Godkin D (2006) Should children's autonomy be respected by telling them of their imminent death? J Med Ethics 32:24-25

25. Kesselheim JC, Johnson J, Joffe S (2010) Ethics consultation in children's hospitals: results from a survey of pediatric clinical ethicists. Pediatrics 125:742-746

26. Larcher V, Hird MF (2002) Withholding and withdrawing neonatal intensive care. Curr Paediatr 12:470-475

27. McGee G, Spanogle JP, Caplan AL, Asch DA (2001) A national study of ethics committees. Am J Bioeth 1:60-64

28. Ramsauer T, Frewer A (2009) Clinical ethics committees and pediatrics. An evaluation of case consultations. Diametros 22:90-104

29. Steinkamp NL, Gordijn B, Ten Have HAMJ (2008) Debating ethical expertise. Kennedy Inst Ethics J 18:173-192

30. Street K, Ashcroft R, Henderson J, Campbell AV (2000) The decision making process regarding the withdrawal or withholding of potential life-saving treatments in a children's hospital. J Med Ethics 26:346-352

31. Taylor HA, McDonald EL, Moon M, Hughes MT, Carrese JA (2009) Teaching ethics to paediatrics residents: the centrality of the therapeutic alliance. Med Educ 43:952-959

32. Vaught W (2008) Autonomy and the rights of minors. Autonomy and human rights in health care. Springer, Dordrecht, pp 111-122

33. Weisleder P (2008) Physicians as healthcare surrogate for terminally ill children. J Med Ethics 34:e8 